\title{
Village néolithique de Klimonas (Ayios Tychonas) - 2018
}

François Briois, Jean-Denis Vigne, Laurence Astruc, Pierre-Antoin

Beauvais, Thomas Cucchi, Benoît Devillers, Bernard Gassin, Matthieu Lebon, Claire Manen, Pantelitsa Mylona, Simon Puaud, Solange Rigaud et Jérôme Robitaille

\section{OpenEdition} Journals

Édition électronique

URL : http://journals.openedition.org/baefe/1695

DOI : $10.4000 /$ baefe. 1695

ISSN : 2732-687X

Éditeur

ResEFE

\section{Référence électronique}

François Briois, Jean-Denis Vigne, Laurence Astruc, Pierre-Antoin Beauvais, Thomas Cucchi, Benoît Devillers, Bernard Gassin, Matthieu Lebon, Claire Manen, Pantelitsa Mylona, Simon Puaud, Solange Rigaud et Jérôme Robitaille, «Village néolithique de Klimonas (Ayios Tychonas) - 2018 » [notice archéologique], Bulletin archéologique des Écoles françaises à l'étranger [En ligne], Chypre, mis en ligne le 26 novembre 2020, consulté le 11 décembre 2020. URL : http://journals.openedition.org/baefe/1695 ; DOI : https://doi.org/10.4000/baefe.1695

Ce document a été généré automatiquement le 11 décembre 2020.

\section{c)}

Le Bulletin archéologique des Écoles françaises à l'étranger est mise à disposition selon les termes de la Licence Creative Commons Attribution - Pas d'Utilisation Commerciale - Pas de Modification 4.0 International. 


\title{
Village néolithique de Klimonas (Ayios Tychonas) - 2018
}

\author{
François Briois, Jean-Denis Vigne, Laurence Astruc, Pierre-Antoin \\ Beauvais, Thomas Cucchi, Benoît Devillers, Bernard Gassin, Matthieu \\ Lebon, Claire Manen, Pantelitsa Mylona, Simon Puaud, Solange Rigaud et \\ Jérôme Robitaille
}

\section{NOTE DE L'AUTEUR}

Autorité nationale présente : Yiannis Violaris (Musée de Limassol)

Numéro de mission : 2018_A_00007

Composition de l'équipe de terrain : L. ASTRUC (Tracéologue, Cnrs Paris), P.-A. BEAUVAIS (lithicien, Université Toulouse 2), T. CUCCHI (archéozoologue, CNRS-Mnhn Paris), B. DEVILLERS (géomorphologue, Univ. Montpellier), B. GASSIN (Tracéologue), M. LEBON (Etude des colorants, Mnhn Paris), S. MADER (spécialiste du macro-outillage, Université Toulouse 2),C. MANEN (Vaisselle de pierre, Cnrs Toulouse), P. MYLONA (géoarchéologie, Mnhn-CNRS Paris), s. PUAUD (Ingénieur en archéométrie, CNRS-Mnhn Paris), S. RIGAUD (spécialiste des parures, CNRS Bordeaux) et J. ROBITAILLE (spécialiste du macro-outillage, Univ. Toulouse II).

L'étude du site s'appuie également sur les compétences scientifiques de A. AVERBOUH (industrie osseuses, CNRS-MNHN Paris), s. BAILON (herpétofaunes, CNRS-MNHN Paris), P. BEAREZ (ichtyofaunes, CNRS-MNHN Paris), C. BENECH (géophysique, CNRS-Univ. Lyon), C. DELHON (phytolithes, CNRS Nice), Y. FRANEL (stratigraphie, INRAP, GO), L. Gourichon (ornithofaune, CNRS Univ. Nice), B. GRATUZE (analyse des obsidiennes, CNRS Orléans), R. HADDAD (approche sociologique, Doc. Nanterre), F. LE MORT (anthropologie, CNRS Lyon), L. MANCA (industrie osseuse, P-Doc Paris), A. QUILES (datations IFAO Le Caire), N. SERRAND (malacologie, INRAP Antilles), A. TABBAGH (géophysique, Univ. PM Curie Paris), S. THIEBAULT (anthracologie, CNRS Paris), O. TOMBRET (datations, CNRS-MNHN Paris), R. TOUQUET (topographie, INRAP IdF), J. WATTEZ (micromorphologie, INRAP IdF), 
G. WILLCOX (carpologie, CNRS Lyon), A. ZAzzo (datations, biogéochimie, CNRS-MNHN Paris).

Partenariats institutionnels : Ministère de l'Europe et des Affaires Étrangères (Mission « Néolithisation-Klimonas »), CNRS, à travers le Site d'Étude en Écologie Globale « Limassol » (SEEG) de l'Institut Écologie et Environnement (INEE), le Muséum national d'Histoire naturelle (Actions thématiques du Muséum), l'UMR 7209, Archéozoologie, archéobotanique : sociétés, pratiques et environnements, l'Institut National de Recherches Archéologiques Préventives (INRAP)

Établissement porteur : École française d'Athènes, Ministère de l'Europe et des Affaires étrangères, CNRS

Remerciements : Nous adressons toute notre gratitude à A. Farnoux, directeur de L'École française d'Athènes, qui nous a fait bénéficier de la base archéologique d'Ayios Tychonas, lieu de traitement et de stockage des matériaux issus de la fouille. Nous remercions également M. Solomidou-Leromynidou, directrice du Département des Antiquités de la République de Chypre qui nous a accordé les autorisations nécessaires et prodigué ses encouragements, et Y. Violaris, directeur du musée de Limassol et responsable du district de Limassol, qui a assuré avec bienveillance le suivi scientifique.

\section{Données scientifiques produites :}

Nouvelles données sur les débuts du Néolithique à Chypre

Agios Tychonas - Klimonas: French Archaeological Mission par le Département des Antiquités de Chypre

Mission archéologique française de Klimonas par l'Institut française de Chypre Programmes « Ayios Tychonas - Klimonas, le plus ancien village chypriote » par l'AIBL

Blog sur Klimonas

\section{Rappels des résultats antérieurs}

1 Le site de Klimonas, localisé sur la commune d'Ayios Thychonas près de Limassol, a été découvert au cours de prospections systématiques conduites vers la fin des années 1980 par Catherine Petit-Aupert dans le cadre de la mission d'Amathonte ${ }^{1}$. L'importance de ce nouvel établissement néolithique a pu être évaluée par des sondages réalisés en $2009^{2}$, puis confirmée au cours des campagnes de fouilles conduites par notre équipe entre 2011 et 2016. La période d'occupation de Klimonas correspond à la fin de la plus ancienne phase du Néolithique précéramique de l'île, datée entre 9100 et 8600 , contemporaine du « Pre-Pottery Neolithic A » récent du continent voisin.

\section{Campagnes 2011-2012 : le bâtiment communautaire du secteur central}

2 Les premières campagnes de de fouilles, réalisées en 2011 et 2012, ont mis en évidence une grande densité de fosses et de structures bâties en terre crue, ainsi qu'au sud-est, les restes très érodés de bâtiments domestiques PPNA (Pre Pottery Neolithic A). La plus remarquable découverte fut un bâtiment parfaitement circulaire, de volume exceptionnel (10 m de diamètre), nommé St 10, dans le secteur central du site (fig. 1). 
Aménagé en fosse sur une hauteur conservée par endroits d'encore un mètre, cet édifice en partie creusé dans le substrat géologique comportait, sur sa périphérie interne, une tranchée servant d'assise à un mur de terre crue plaqué contre l'encaissant ${ }^{3}$. Un dispositif d'entrée ouvre vers le nord-est. Cette grande construction mise en en évidence à Klimonas correspond à une catégorie d'édifices connus en Turquie, Syrie, Irak, Palestine et Jordanie, qualifiés, en raison de leur volume et de leur originalité, de "bâtiments communautaires". La comparaison avec les quelques monuments levantins connus démontre que, dès les débuts du Néolithique, Chypre était partie prenante d'une même « ambiance culturelle » qui affectait alors le LevantNord.

\section{Campagnes 2014-2015 : Extension de la fouille et délimitation du village}

3 Suite à une série de prospections géophysiques ${ }^{4}$ suivie d'une campagne de vastes décapages mécaniques, la fouille a été implantée sur deux secteurs éloignés de quelques dizaines de mètre du secteur central :

- Dans le secteur $\mathrm{F}$, situé à la limite nord-ouest de l'établissement, un bâtiment de forme circulaire de $6 \mathrm{~m}$ de diamètre (St 800), particulièrement bien conservé, a pu offrir un premier exemple d'architecture domestique (fig. 2). La moitié nord a été entaillée dans le versant, et la partie sud repose sur un remblai de nivellement. Le bâtiment était pourvu d'un sol enduit soigné et d'un ensemble complexe d'aménagements, notamment un mur périphérique de bauge implanté dans une tranchée de fondation, de nombreux poteaux et piquets, de petites tranchées internes délimitant des banquettes ou des cloisons et des caches comportant des dépôts d'objets remarquables.

- Le secteur B, localisé au sud, a été exploré sous la forme de sondages qui ont révélé un vaste complexe stratigraphique contenant de riches niveaux d'occupation PPNA.

Ces résultats, associés à ceux des campagnes antérieures, permettaient d'identifier les limites occidentale et méridionale du village néolithique et d'estimer sa surface à un demi-hectare ${ }^{5}$. 
Fig. 2. Le bâtiment St 800 dans le secteur F à la fin de la campagne 2015.

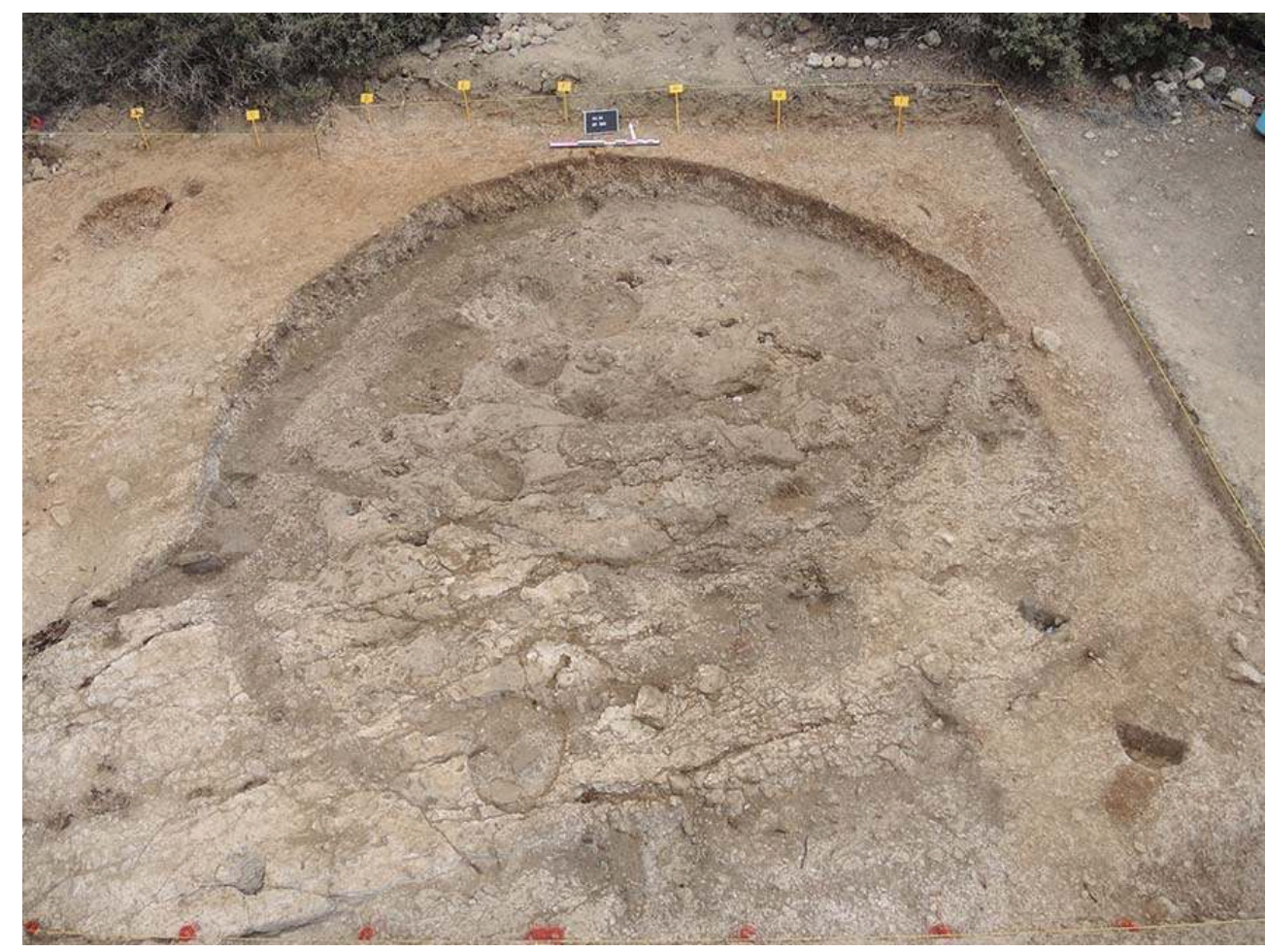

J.-D. Vigne.

\section{Campagne 2016 : exploration de l'extension sud du village}

Le dégagement extensif réalisé sur le secteur $\mathrm{B}$, correspondant à la limite sud du village néolithique, a permis la fouille de 21 restes de bâtiments de plan circulaire et aux diamètres estimés entre 3,5 et 7 mètres (fig. 3). Tous ces bâtiments sont délimités par une tranchée de fondation curviligne, peu profonde, dans laquelle était implanté un mur de terre crue, parfois armé de poteaux de bois. Les tranchées de fondation et les sols délimitent des terrasses sub-horizontales encochées dans le versant, selon un modèle comparable à celui du bâtiment du secteur $\mathrm{F}$ (St 800). Certaines de ces terrasses ont été réutilisées pour implanter un second, voire un troisième bâtiment plus petit que le précédent. Les sols sont percés de nombreux trous de piquets ou de poteaux et de diverses fosses dont certaines contenaient des caches d'objets. On observe une certaine disparité de tailles, d'architecture et d'équipement interne : des bâtiments de moins de $10 \mathrm{~m}^{2}$, au contour moins régulier, aux murs moins bien étayés et abritant parfois un grand bloc de pierre à profonde cupule piquetée peuvent être assimilé à des abris à vocation artisanale, notamment pour le traitement des récoltes; les édifices clairement domestiques sont plus grands (plus de $12 \mathrm{~m}^{2}$ ), étayés à l'amont et toujours pourvus d'une grande fosse-foyer. À l'issue de cette cinquième campagne de fouille, il était dès lors possible de donner un assez bon descriptif spatial et temporel du village de Klimonas (fig. 4). Avec une surface conservée de $5600 \mathrm{~m}^{2}$, le site se place dans la moyenne haute des villages PPNA du Levant, et, de par son insularité, constitue donc un site majeur pour cette période. 
Fig. 3. L'extension sud du village néolithique dans le secteur B à la fin de la campagne 2016.

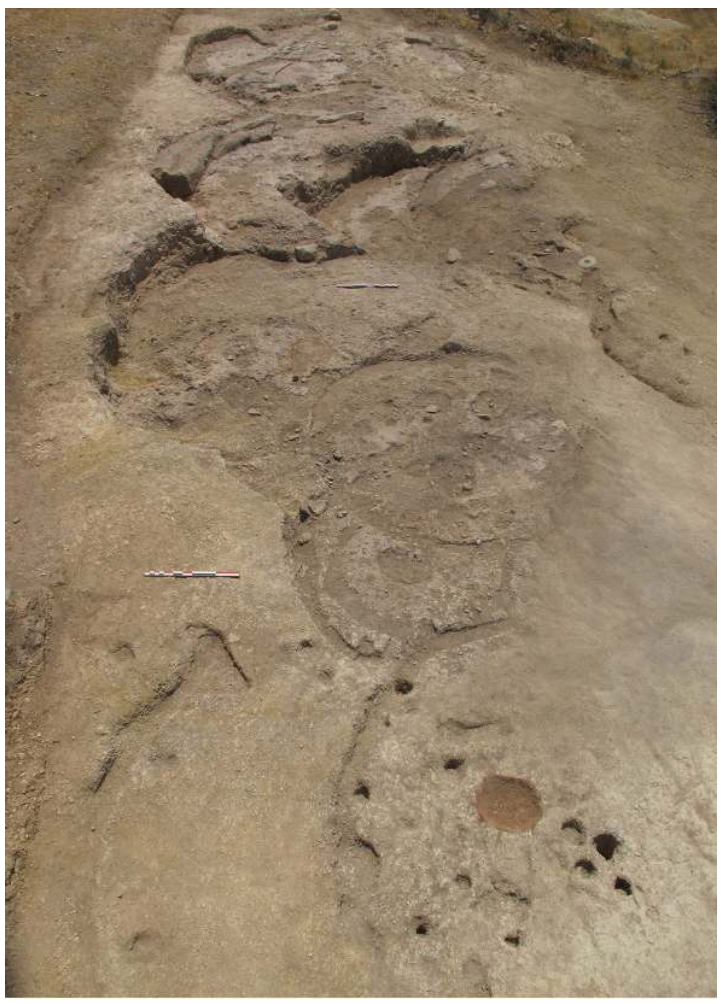

J.-D. Vigne.

Fig. 4. Plan des secteurs fouillés à Klimonas depuis 2011 et localisation des bâtiments PPNA de la partie explorée du village néolithique.

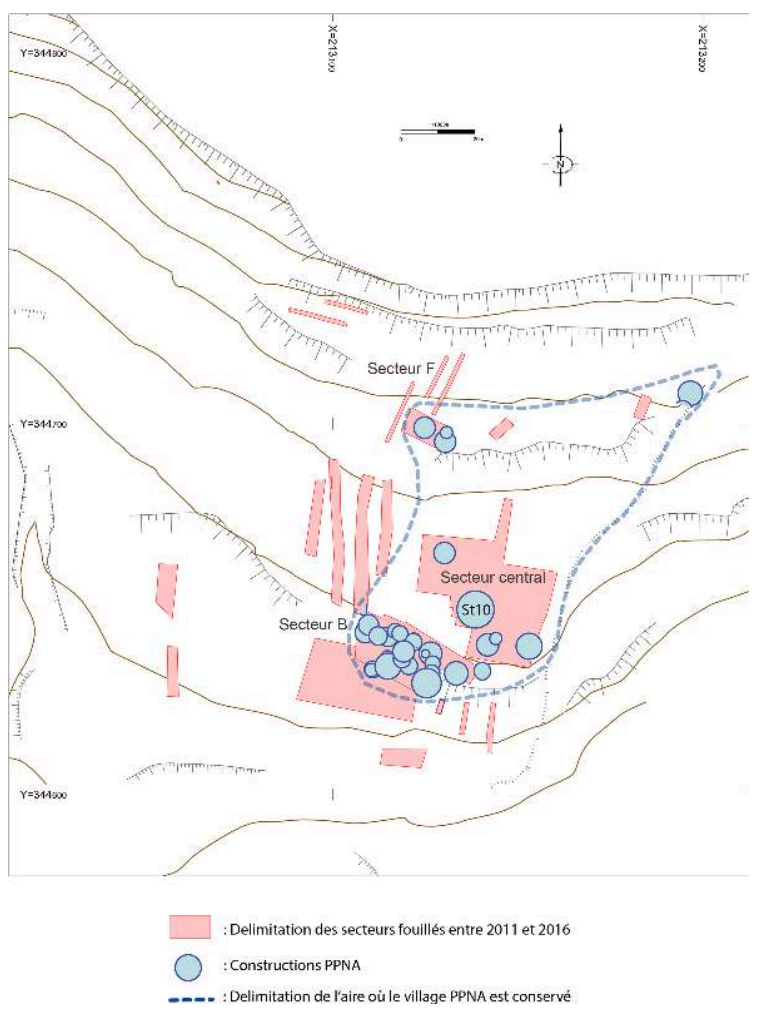

Modèle numérique de terrain : R. Touquet, INRAP. 
La campagne 2017 a exclusivement été consacrée à une mission d'étude des mobiliers et des restes bio-archéologiques de Klimonas. Ce travail a pu être en grande partie réalisé pour les séries lithiques taillées, le macro-outillage et les objets de parures qui constituaient, de toute évidence, une priorité majeure compte tenu de l'extrême abondance des pièces à étudier. Cette urgence était accrue par la double nécessité d'identifier les séries sur lesquelles devaient être engagées les analyses et de préparer les mobiliers pour lesquels l'étude était reportée à l'année suivante.

\section{Mission d'étude sur les mobiliers et les restes bio- archéologiques}

7 La campagne d'étude 2018 a pu bénéficier du travail de fond réalisé au cours de la mission précédente pour se consacrer pleinement au traitement des divers mobiliers dont l'étude était à poursuivre ou, dans le meilleur des cas, à finaliser. Les travaux réalisés en mai 2018 ont ainsi portés sur :

- La poursuite des analyses technologiques et typologiques des industries de pierre taillée par F. Briois et P.-A. Beauvais,

- La réalisation d'une étude techno-fonctionnelle à partir d'un échantillonnage d'outils lithiques, dont l'achèvement est prévu en 2019, par L. Astruc et B. Gassin,

- L'achèvement du traitement et de l'analyse du macro-outillage par J. Robitaille,

- L'enregistrement et l'analyse des objets de parure en pierre et en coquille fossile par S. Rigaud,

- Le traitement des colorants (inventaire, enregistrement et sélection d'un échantillonnage pour analyses par Matthieu Lebon et Simon Puaud,

- L'étude archéozoologique et l'enregistrement de tous les restes de faune des campagnes 2015 et 2016 par J.-D. Vigne,

- La poursuite et l'achèvement de l'enregistrement photographique 2D des restes dentaires et 3D des calcanéus de sanglier, réalisé par T. Cucchi, en vue de l'analyse morphométrique à venir.

\section{Étude des outillages lithiques}

François Briois et Pierre-Antoine Beauvais

Un des principaux objectifs de la mission 2018 a été l'achèvement du traitement des séries du secteur B, notamment toutes celles qui provenaient des structures (trous de poteau, tranchées de fondation, foyers, fosses, caches et dépôts), dont le total s'élève à 11357 pièces. Ce travail a été complété par l'achèvement de l'étude des pointes de projectile du site, par l'enregistrement d'une importante série d'outils du fonds commun, notamment les lames à lustré d'usage, et l'étude de la petite collection de pièces en obsidienne. Les nucléus à lames ont fait l'objet d'une étude technologique spécifique réalisée par P.-A. Beauvais et complétée par la réalisation d'illustrations (photos et dessins).

9 L'industrie est à forte composante laminaire et toutes les étapes de plusieurs chaînes opératoires ont pu être étudiées en détail à partir des produits de débitage, des déchets à techniques significatifs et d'un grand nombre de nucléus. Leur étude a permis de déterminer les différents modes de production de lames qui se caractérisent principalement à Klimonas par l'exploitation de nucléus coniques ou pyramidaux à 
débitage enveloppant ou semi-enveloppant (fig. 5). Ces nucléus sont destinés à produire des lames à profil rectiligne et à nervures convergentes destinées à la fabrication des pointes de projectiles et des armatures de faucille qui constituent les deux catégories d'outils essentiels en rapport avec l'économie de subsistance du site fondée sur les activités cynégétiques et la récolte de céréales.

Fig. 5. Fiche descriptive et schéma diacritique d'un nucléus à lames.

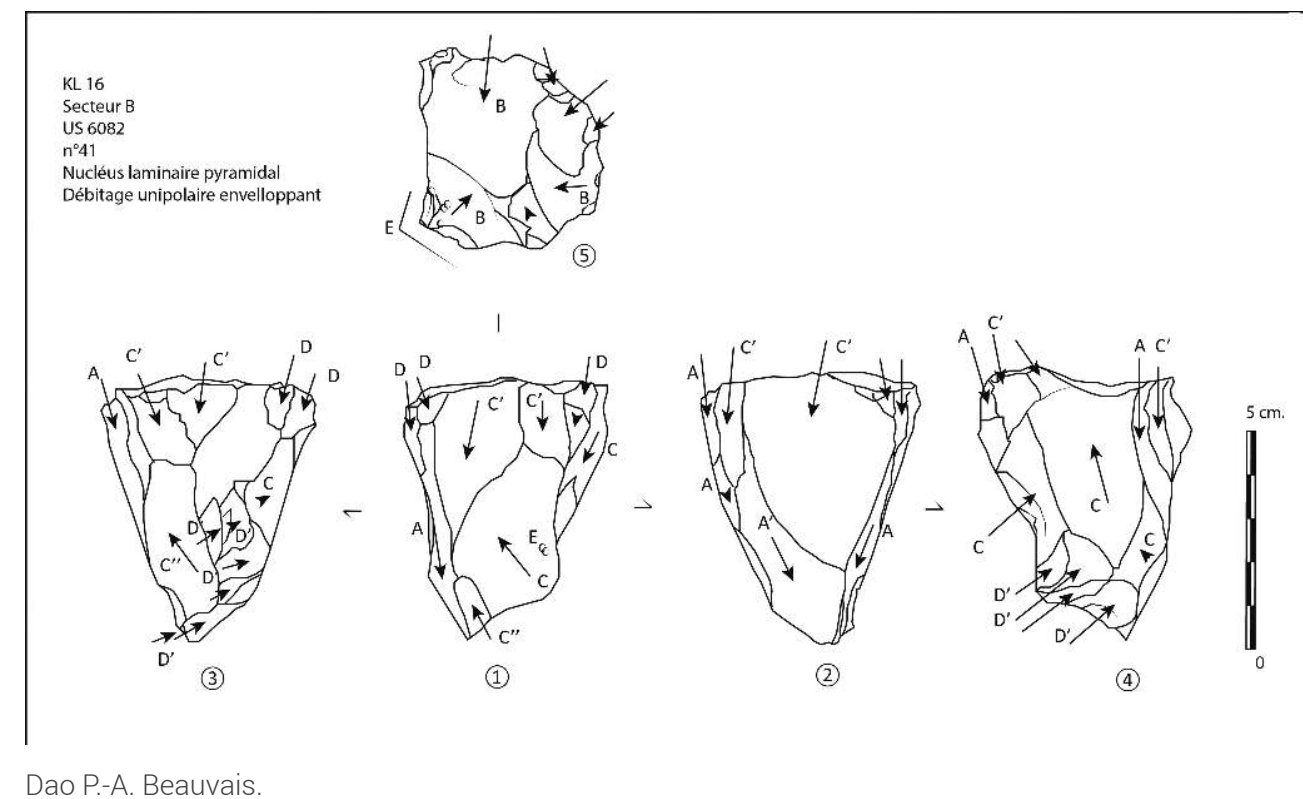

\section{Analyse fonctionnelle des outillages}

Laurence Astruc et Bernard Gassin

L'étude techno-fonctionnelle a porté sur l'industrie de l'unité stratigraphique 10.6, correspondant à l'un des multiples niveaux d'occupation et de rejet présents au sein du bâtiment communautaire. Cet assemblage a été choisi en raison de sa cohérence, de sa très bonne conservation et du très grand nombre d'outils. Sur le plan méthodologique, les analyses ont été réalisées à l'aide de binoculaires (jusqu'à 60x de grossissement, MCS-10 et MOD566) et d'un microscope métallographique (avec des objectifs de 50x, 100x et 200x, Olympus BH2-UMA). L'échantillonnage de 686 pièces analysées, incluant des produits bruts de débitage et des pièces transformées par la retouche, montre un taux d'utilisation faiblement élevé. Les outillages ont globalement été employés pour des travaux de faible ampleur avec un usage bref et un rejet rapide. Quelques pièces mettent cependant en lumière des utilisations multiples avec de rares cas de recyclages. La large gamme de modes d'action et de matières travaillées est en rapport avec les activités de boucherie, le travail de l'os, de la peau, de coquilles, de pierres tendres et de matières végétales rigides. Des petits travaux de raclages de futs végétaux sont les plus fréquents : ils correspondent à des finitions de petits objets en bois comme des fûts de flèches ou d'autres petits mobiliers. Les résultats obtenus à Klimonas sont concordants avec ceux qui ont été mis en évidence à Shillourokambos et Khirokitia mais avec un taux d'utilisation inférieur. 
Fig. 6. Burin utilisé sur l'un de ses bords bruts pour un sciage de bois.
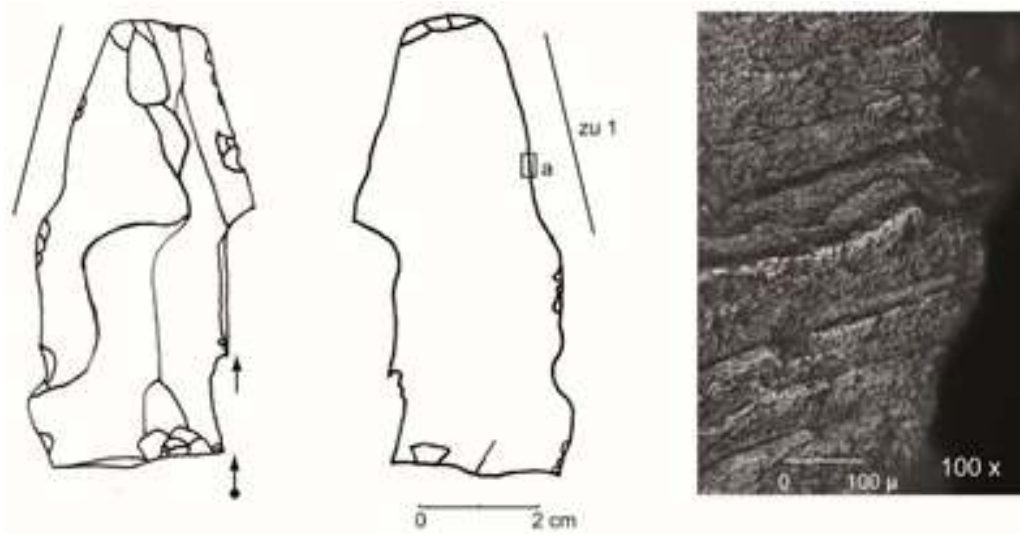

Dao P.-A. Beauvais.

\section{Étude du macro-outillage}

Jérôme Robitaille

11 L'étude du macro-outillage de Klimonas a porté sur un effectif total de 942 pièces distribuées dans 23 catégories. L'objectif a été d'achever l'enregistrement des caractères descriptifs, commencé en 2017 (matériau, dimensions, morphologie, localisation et description des surfaces actives, traces de mise en forme et restes de résidus) et d'en tirer un bilan. Le large spectre d'outils est composé en majeure partie d'outils utilisés en percussion lancée (39\%), d'instruments de mouture, qui regroupent les meules et les molettes ( $23 \%$ ) et de retouchoirs/compresseurs (12\%).

Les matériaux employés correspondent globalement à des roches tenaces d'origine magmatique qui ont été sélectionnées dans les formations fluviatiles régionales en respectant les critères précis de poids, de volume, de morphologie et de qualité du grain nécessaires à la fabrication et au fonctionnement des divers instruments. Cette sélection confère une forme de normalisation à certains types d'outils, comme dans le cas des bouchardes ou des retouchoirs/compresseurs, qui n'ont pas fait l'objet d'une mise en forme préalable. Les instruments de mouture et les maillets à gorges sont les seuls outils à avoir fait l'objet d'une véritable chaîne opératoire de fabrication, transformant la morphologie initiale du bloc. Les outils connaissent souvent plusieurs cycles d'utilisation et d'entretien et certains ont eu plusieurs fonctions, comme dans le cas des molettes à grain utilisées également pour le broyage d'ocre (fig. 7). Il existe aussi des cas de réemploi d'outils usagés ou cassés pour d'autres fonctions, c'est le cas d'anciennes molettes réutilisées comme enclumes. 
Fig. 7. A : Molette ocrée ; B : Molette ; C : Plaquette de calcaire ocrée ; D : enclume sur bloc calcaire.

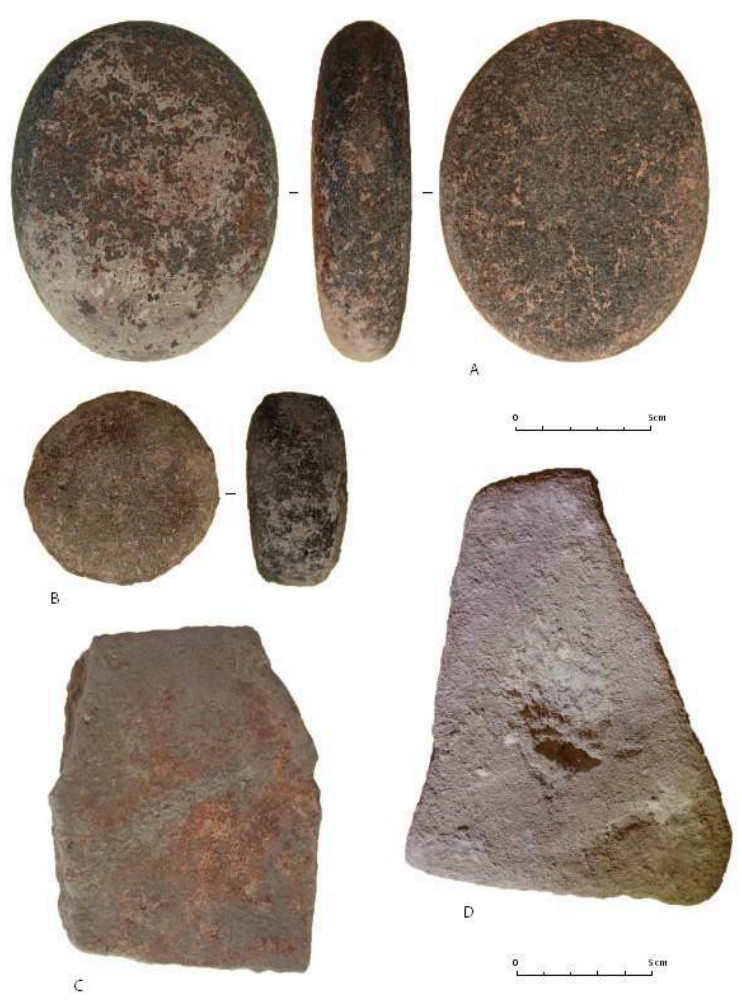

J.-D. Vigne.

\section{La vaisselle de pierre}

Claire Manen

L'objectif était l'analyse des récipients de pierre découverts lors des différentes campagnes de fouille de Kimonas. Ces pièces ont fait l'objet d'un inventaire et d'une couverture photographique qui a porté sur 105 individus au total.

Sur la base des critères classificatoires établis pour le site de Shillourokambos ${ }^{6}$, 58 éléments peuvent être rapportés à une catégorie morpho-fonctionnelle spécifique :

- des ustensiles plats et ouverts (plats et coupelles distingués sur des critères de taille et donc de maniabilité probablement destinés à travailler et/ou présenter des aliments ou tout autre matière non alimentaire (pigments, onguents ?...);

- des récipients ouverts plus profonds, mais dont la taille permet la manipulation (bols à profil hémisphérique) ;

- des récipients ouverts profonds et surtout de grande taille dont la manipulation est plus difficile (bassins, bol-mortiers) ;

- des pièces plus énigmatiques, regroupées sous l'appellation « godet ».

Le point de comparaison le plus immédiat est celui fourni par le site contemporain d'Asprokremmos ${ }^{7}$ où trois vases complets en calcaire crayeux ont été découverts. L'un de ces vases contenait des restes d'ocre témoignant de l'intégration de la vaisselle de pierre dans la sphère de traitement de matières non alimentaires. Sur le continent, les sites permanents ou semi permanents du PPNA ('Ain Darat, Jericho, Netiv Hagdud, Gilgal $\mathrm{I}, . .$.$) ne livrent également que très peu de vaisselle { }^{8}$. Le corpus de Klimonas s'intègre donc dans les normes de production du PPNA où il n'existe pas de recherche 
systématique de symétrie et où les formats sont simples (bols ou bassins hémisphériques et plats majoritairement). La présence de pigments dans plusieurs récipients constitue un fait remarquable, renforcé par le parallèle avec Asprokremmos.

\section{Les objets de parures de Klimonas}

Solange Rigaud de parure qui compte 1443 artefacts. L'ensemble des pièces a été soumis à l'identification taxinomique et de matière première et il a fait l'objet de mesures et d'observations technologiques et tracéologiques complémentaires. Les objets de parures en matière minérale sont presque exclusivement en picrolite, roche verte tendre de la vallée du Kouris, accessible à une vingtaine de kilomètres à l'ouest de Klimonas. Une prospection sur les sources a permis de procéder à un échantillonnage et d'observer les modalités de collecte et de sélection des galets naturels effectuées par les villageois de Klimonas. Les objets en coquille marine utilisent un spectre malacologique très diversifié au sein duquel trois espèces sont largement surreprésentées: les dentales, les spondyles et les valves de Glycymeris sp. L'approvisionnement, sans doute mixte, combine un prélèvement sur les plages marines contemporaines de l'occupation du site et sur des gittes fossilifères. L'industrie en spondyle fossiles de grande taille, qui fait appel à une chaîne opératoire complexe pour la fabrication de perles tubulaires, est à ce jour la plus ancienne actuellement documentée dans le bassin méditerranéen. La chaine opératoire des objets en matière dure minérale est entièrement réalisée sur le site. Elle adopte un mode opératoire très simple et les objets produits n'ont aucune standardisation de forme ni de procédé technique.

\section{Étude des matières colorantes}

Matthieu Lebon et Simon Puaud

17 La fouille du site de Klimonas a permis de mettre au jour de nombreux fragments de roches de couleur verte et des blocs de matières colorantes rouges, jaunes et noires qui ont été soumis à une analyse minéralogique dans le but de déterminer leur origine. Un corpus de 30 échantillons composé de 14 éléments de roches dures de couleur verte et 16 de matières colorantes (rouge, jaune et noire) a fait l'objet d'une analyse par diffraction des rayons X (DRX) et par spectrométrie de fluorescence des rayons X (XRF). Des prospections de terrain ont été conduites dans le but de rechercher les sources géologiques potentielles et les gîtes d'où ces matériaux auraient pu être extraits. Les objectifs se sont limités aux matières colorantes rouge, jaune et noir dans l'environnement du site de Klimonas, et à la prospection systématique de la vallée du Kouris, sur le flanc sud du Troodos, pour ce qui est des roches vertes.

\section{La faune de vertébrés}

Jean-Denis Vigne, Thomas Cucchi

La campagne d'étude a permis d'achever la révision de la totalité des restes de faune dont les effectifs s'élèvent désormais à 15983 restes, dont 9496 identifiés. Les travaux ont également été consacrés à la mise au point d'une grille d'analyse taphonomique 
puis de procéder à l'enregistrement exhaustif des données des secteurs $\mathrm{B}$ et $\mathrm{F}$. Dans ces deux secteurs, les résultats sont semblables à ceux du secteur central: le sanglier chypriote domine à $96,6 \%$, devant les oiseaux $(1,8 \%)$, les carnivores $(0,4 \%)$, les crabes d'eau douce $(0,2 \%)$ et la microfaune (souris, herpétofaune). À cette occasion toutes les pièces susceptibles de l'être ont été mesurées et les derniers restes relatifs à l'industrie osseuse, ainsi que les ossements d'oiseaux, de reptiles et d'amphibiens ont été réunis en vue de l'achèvement de l'étude des séries correspondantes placées sous la responsabilité respective de A. Averbouh et L. Manca (AASPE), L. Gourichon (CEPAM) et $\mathrm{S}$. Bailon (AASPE). Toutes les séries dentaires de suinés des secteurs $\mathrm{B}$ et $\mathrm{F}$ ont par ailleurs fait l'objet d'une estimation d'âge en vue de l'établissement des profils d'abattage. Pour ces deux secteurs, comme pour le secteur central (St10), chaque rangée dentaire ou dent jugale isolée mesurable a été identifiée par un numéro d'inventaire qui permet de disposer conjointement de ses mensurations linéaires, de son âge et de ses caractéristiques de morphométrie géométrique. La campagne d'étude a par ailleurs permis de reprendre en détail les identifications de chaque pièce de carnivore (37 restes de chien, un de chat) et d'engager une réflexion sur leur distribution spatiale, très proche de celle de la quinzaine de restes humains trouvés en très grande majorité dans le bâtiment communautaire St10. T. Cucchi a numérisé une dizaine de calcanéus en vue de l'analyse morphométrique surfacique.

\section{BIBLIOGRAPHIE}

BENECH, TABBAGH, VIGNE 2017

Christophe Benech, Alain Tabbagh, Jean-Denis Vigne, «Étude par prospections magnétique et électromagnétique du site de Klimonas (Chypre) », in Jean-Denis Vigne, François Briois et Margareta Tengberg (éd.), Nouvelles données sur les débuts du Néolithique à Chypre, Paris, SPF, séances en ligne, 9, 2017, p. 79-94.

BRIOIS, AUPERT, PECHOUX 2005

François Briois, Catherine Aupert, Pierre-Yves Pechoux, Histoire des campagnes d'Amathonte 1. L'occupation du sol au Néolithique, Athènes, EFA, Études chypriotes 16, 2005.

BRIOIS, VIGNE, GUILAINE, FRANEL 2018

François Briois, Jean-Denis Vigne, Jean Guilaine, Yodrik Franel, « Ayios Tychonas - Klimonas », BCH 139-140, 2015-2016, p. 1022-1027.

MANEN 2011

Claire Manen, « La vaisselle de pierre », in Jean Guilaine, François Briois et Jean-Denis Vigne (éd.), Shillourokambos. Un établissement néolithique pré-céramique à Chypre. Les fouilles du secteur 1, Paris, Athènes, Errance, EFA, 2011, p. 769-787.

MANEN 2017

Claire Manen, « Manufacturing and use of the stone vessels from PPN Shillourokambos in the context of Cypriot and Near Eastern PPN stone vessel production », in Jean-Denis Vigne, François 
Briois, Margareta Tengberg (éd.), Nouvelles données sur les débuts du Néolithique à Chypre, Paris, SPF, séances en ligne, 9, 2017, p. 167-182.

MCCARTNEY 2017

Carole McCartney, «Ayia Varvara Asprokremnos : a late PPNA specialised site on Cyprus », in Jean-Denis Vigne, François Briois, Margareta Tengberg (éd.), Nouvelles données sur les débuts du Néolithique à Chypre, Paris, SPF, séances en ligne, 9, 2017, p. 47-58.

ROSEMBERG 2008

Danny Rosemberg, « Serving Meals making a Home. The PPNA Limestone Vessel Industry of the Southern Levant and its Importance to the Neolithic Revolution », Paléorient 34.1, 2008, p. 23-32. VIGNE et al. 2011

Jean-Denis Vigne et alii, « Preliminary data on a new early Pre-Pottery Neolithic site on Cyprus (Ayios Tychonas - Klimonas, ca. 9000 cal. BC) », Néo-Lithics 11.1, 2011, p. 3-18.

VIGNE et al. 2012

Jean-Denis Vigne et alii, « The first wave of cultivators spread to Cyprus at least 10,600 years ago », PNAS 102, n² 22, 2012, p. 8445-8449.

VIGNE et al. 2017

Jean-Denis Vigne et alii, « Klimonas, a late PPNA hunter-cultivator village in Cyprus: new results, in Jean-Denis Vigne, François Briois et Margareta Tengberg (éd.), Nouvelles données sur les débuts du Néolithique à Chypre, Paris, SPF, séances en ligne, 9, 2017, p. 21-46.

\section{NOTES}

1. BRIOIS, AUPERT, PECHOUX 2005

2. VIGNE et al. 2011.

3. VIGNE et al. 2012 et 2017.

4. BENECH, TABBAGH, VIGNE 2017.

5. BRIOIS, VIGNE, GUILAINE, FRANEL 2018.

6. Manen 2011 et 2017.

7. Mc Cartney 2017.

8. Rosenberg 2008. 
INDEX

Thèmes : EFA

Année de l'opération : 2018

lieux https://ark.frantiq.fr/ark:/26678/pcrtS4MSAHu2F3

chronologie https://ark.frantiq.fr/ark:/26678/pcrtH8P95EucZz

sujets https://ark.frantiq.fr/ark:/26678/pcrtPEkp3Yydb2, https://ark.frantiq.fr/ark:/26678/ pcrtERsRub9G3c, 26678/pcrtT2H2KjqBcU, 26678/pcrtIMsuG0RWTs, https://ark.frantiq.fr/ark:/ 26678/pcrtUDM5NvfJZ1, https://ark.frantiq.fr/ark:/26678/pcrtmkRNd3ikl4, https:// ark.frantiq.fr/ark:/26678/pcrtbfqVEeganP, https://ark.frantiq.fr/ark:/26678/pcrtNBrnOdttU9

\section{AUTEURS}

\section{FRANÇOIS BRIOIS}

UMR 5608 « Travaux et Recherches Archéologiques sur les Cultures, les Espaces et les Sociétés »

\section{JEAN-DENIS VIGNE}

UMR 7209 « Archéozoologie, archéobotanique : sociétés, pratiques et environnements »

\section{LAURENCE ASTRUC}

UMR 7041 ArScAn

\section{PIERRE-ANTOIN BEAUVAIS}

doctorant université Toulouse 2),

\section{THOMAS CUCCHI}

UMR 7209, CNRS-MNHN

\section{BENOÎT DEVILLERS}

Université Montpellier 3

BERNARD GASSIN

Éducation Nationale

MATTHIEU LEBON

UMR 7194, MNHN-CNRS

\section{CLAIRE MANEN}

UMR 5608 TRACES

\section{PANTELITSA MYLONA}

doctorante MNHN

SIMON PUAUD

UMR 7194,CNRS-MNHN 
SOLANGE RIGAUD

UMR 5199 PACEA

JÉRÔME ROBITAILLE

doctorant EHESS 